\title{
Matching and Transport of Beams in a Continuously Twisted Quadrupole Channel
}

\author{
Tiefenback, M. G., J. P. Lidestri, V. L. Bailey, Jr, and S. D. Putnam \\ Pulse Sciences, Inc., 600 McCormick St., San Leandro, CA 94577
}

\begin{abstract}
Twisted quadrupole (stellarator) focussing can reduce dispersion in the bends of a recirculating accelerator, such as the Spiral Line Induction Accelerator[1] (SLIA) under development at Pulse Sciences. Envelope oscillations caused by guide field/stellarator transitions add free energy to the beam which can couple into the beam emittance. This effect can be reduced by phase space matching of the beam at the transitions. We present experimental results on the coupled 4-D problem of envelope matching into a straight helical quadrupole channel, compare these results against envelope code predictions, and discuss expected benefits from such matching in a few-turn accelerator system.
\end{abstract}

\section{Introduction}

Helical quadrupole focussing[2] was added by Roberson, et al.[3], to the beam dynamics of a modified (toroidal guide field) betatron, greatly enhancing the energy mismatch tolerance over that of normal and modified betatrons. Putnam[1] adapted this focussing to an open-ended spiral geometry for multiple passes of high-current beams through common acceleration cavities, sidestepping closedorbit injection and extraction problems. For practical reasons, quadrupole focussing is incorporated in the SLIA only within the bends. The chromatically varying envelope mismatch at uncompensated stellarator/guide field transitions can significantly increase the time-integrated transverse emittance of the beam. Envelope mismatches can also drive local emittance growth[4,5]. Mismatch mode coherence can be lost if particle oscillation frequencies vary. In the emittance-dominated limit the matched beam size scales as the square root of the emittance, and a $10 \%$ additional radial excursion of the beam can result in a $20 \%$ increase in effective emittance. Of particular concern is the relative phase of successive mismatches.

\section{Phase space structure}

The evolution of real beams san be modeled by the K-V distribution[6], in which unrealistic $x^{\prime}-y^{\prime}$ correlations enforce uniformity of the beam current density. Practical beam matching involves providing the proper $x-y$ profile and $x-x^{\prime} / y-y^{\prime}$ focussing for the beam emittance. Analytical twisted quadrupole equilibria[2], including a guide

*Work supported by DARPA under Order No. 4395, Amendment 90 , and by the Navy under document N00039991 WXDZ002; monitored by the Naval Surface Warfare Center

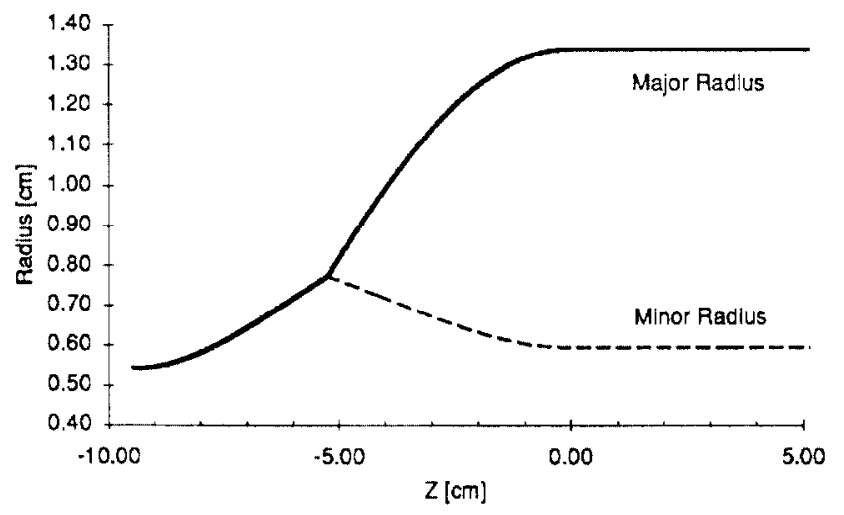

Figure 1: Matching transition between solenoid and stellarator focussing, a thin-lens existence proof.

$\begin{array}{cccc}\text { Current: } & 10 \mathrm{kA} & \text { Energy: } & 3 \mathrm{MeV} \\ 4 \epsilon_{\mathrm{n}, \mathrm{RMS}} & 158 \mathrm{~cm} \mathrm{mrad} & B_{z} & 4666 \mathrm{G} \\ R_{\text {waist }} & 0.5424 \mathrm{~cm} & z_{\text {waist }} & -9.24 \mathrm{~cm} \\ \text { Stel. pitch } & 31.42 \mathrm{~cm} & \text { Gradient } & 405.5 \mathrm{G} / \mathrm{cm} \\ \text { Lens 1 }(\mathrm{z}, \mathrm{G}, \theta) & -5.245 \mathrm{~cm} & 1683 \mathrm{G} & 29.5^{\circ} \\ \text { Lens 2 }(\mathrm{z}, \mathrm{G}, \theta) & 0 \mathrm{~cm} & 10.52 \mathrm{G} & 45.0^{\circ}\end{array}$

Table 1: Thin lens matching example parameters

field[7], exist as $x-y$ coupled generalizations of the $\mathrm{K}-\mathrm{V}$ model. These equilibria are elliptical in real space and have skew $x-y^{\prime} / y-x^{\prime}$ focussing[2] and an overall rotation determined by the guide field and the total canonical angular momentum of the bean. Chernin[8] derived moment evolution equations for coupled $x-y$ systems and provided us with Fortran coding to integrate the equations. This program was used to design a thin lens doublet to match a round, field-free beam into a stellarator channel[9] (Figure 1), showing the feasibility of nonadiabatic stellarator matching. The relevant parameters arc shown in Table 1. The stellarator mouth is at $z=0 \mathrm{~cm}$. Consider the inverse problem of matching from a stellarator equilibrium into a solenoid. Select a quadrupole lens strength and orientation near the mouth of the stellarator to force the beam profile to be circular at some point in the following guide field (for many equilibria, no lens is necessary). At the point of roundness, place a thin quadrupole with strength and orientation chosen to zero the skew correlations of the beam and equalize the $x-x^{\prime} / y-y^{\prime}$ correlations. The radial oscillations can be controlled by adjusting the $B_{z}(z)$ distribution. For thick lenses, the cyclotron motion becomes important. 


\section{Experimental setup}

The experimental geometry is linear, referenced to the cathode at $\mathrm{z}=\mathbf{0} \mathrm{cm}$. Two-layer solenoid coils (average radii of $3.82 \mathrm{~cm}$ and $4.47 \mathrm{~cm}$ ) span from $37.8 \mathrm{~cm}$ to $80.0 \mathrm{~cm}$ for the transition section (central field $1216 \mathrm{G}$ ) and from $87.1 \mathrm{~cm}$ to $343.7 \mathrm{~cm}$ (central field $1476 \mathrm{G}$ ) for the stellarator section. Two loop coils of average radius $8.3 \mathrm{~cm}$ are used to adjust the $B_{z}(z)$ distribution. One is centered at $82.8 \mathrm{~cm}$, with central field $876 \mathrm{G}$ to compensate for the solenoid interruption, and the other is centered at $49.3 \mathrm{~cm}$, with central field $-516 \mathrm{G}$ to adjust the input beam envelope. Quadrupoles are centered at $64.3 \mathrm{~cm}$ (peak gradient $57.5 \mathrm{G} / \mathrm{cm}$, focussing in a plane $48.2^{\circ}$ clockwise from vertical) and $75.3 \mathrm{~cm}$ (peak gradient $92.2 \mathrm{G} / \mathrm{cm}$, focussing in a plane $1.6^{\circ}$ degrees clockwise from vertical), of effective length $\left(\int B^{\prime}(z) d z / B_{\max }^{\prime}\right) 5.5 \mathrm{~cm}$. A stellarator coil (righthanded, average radius $5.24 \mathrm{~cm}$ and pitch length $62.8 \mathrm{~cm}$ ) spans $89.3 \mathrm{~cm}$ to $340.6 \mathrm{~cm}$, oriented so that at its entrance the beam is defocussed in the vertical plane. The input beam passes through a waist of radius $4.7 \mathrm{~mm}$ at $\mathrm{z}=48.4$ $\mathrm{cm}$, at a guide field of 610 gauss. The beam energy is 800 $\mathrm{keV}$, the current is 195 ampcres, and the timc-integrated normalized emittance is measured to be approximately 31 $\pi \mathrm{cm}$ mrad, using Cherenkov imaging of beamlets from a pinhole array.

The long magnet is an analogue of the 0.8 meter major radius stellarator bend currently in fabrication at Pulse Sciences, Inc., for the proof of concept experiment. The "straight bend" enabled meaningful physics measurements in energy scaled experiments, and comparison of the beam dynamics in a straight geometry with that in the bend should aid us in understanding toroidal effects. The parameters of our beam were chosen to model the $5.0 \mathrm{MeV}$, $10 \mathrm{kA}$ beam expected after the first induction stage of the SLIA at Pulse Sciences. In the anticipated $10 \mathrm{kA}$ system, the space-charge term in the envelope equation is much smaller than the emittance term (emittance proportional to the square of the beam size). However, the emittance is low cnough in these experiments that space-charge is comparable in importance to the emittance.

\section{Results}

Our prinary measurement technique is open-shutter photography of a Cherenkov convertor. A description is given in the paper by Lidestri, et al. [10], at this conference. The images are digitized with a high-resolution scanner, over a range of film exposure calibratcd against mini-Faraday cup current density measurements for a dynamic range greater than 10:1. The beam radii shown here are edge radii, rather than RMS radii. We have placed a small-diameter beam st op on the front surface of the Cherenkov convertor near the center of the beam. An intensity contour is chosen surrounding the stop, of the same diameter. The intensity contour at the same level, surrounding the beam, is used to define the beam size. This protocol, while well-defined, does not yield the RMS beam profile. (We are currently working on an RMS reduction of the beam parameters.)

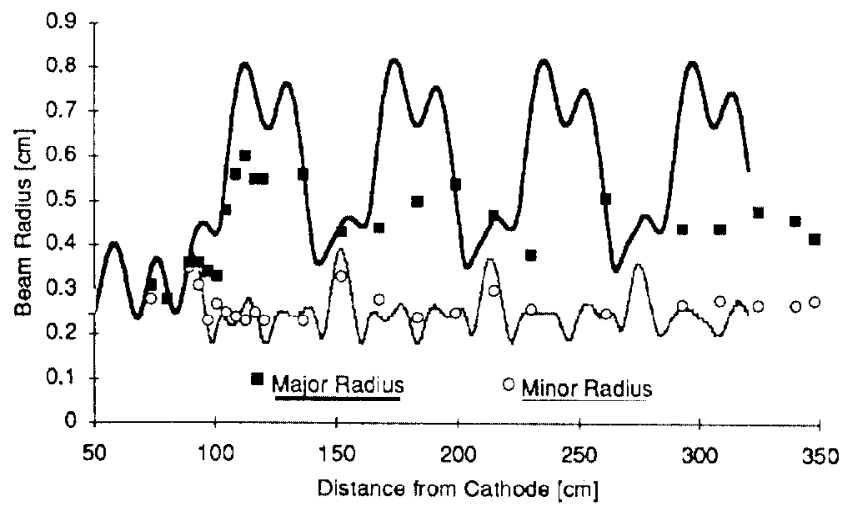

Figure 2: Beam envelope (experimental and calculated) along the channel without matching elements.

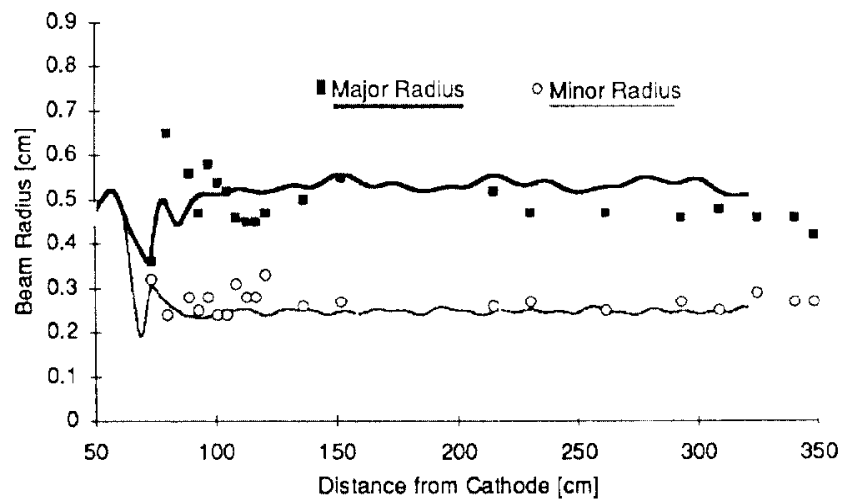

Figure 3: Beam envelope (experimental and calculated) along the channel for the nominal matched configuration of the text.

The matching fields were chosen using the measured edge radii as estimates of the RMS beam radii, and require experimental tuning.

We measured the beam envelope for the nominally matched configuration listed above, as well as for an unmatched case with neither the discrete quadrupoles nor the loop coil at $49.3 \mathrm{~cm}$, and with a field strength in the transition solenoid of $1515 \mathrm{G}$. The unmatched results are shown in Fig. 2. The continuous lines show the calculated envelope. The measured major radius is systematically lower than the calculated value, which may be due to a systematic deviation of our measured edge radius from the RMS radius or to errors in the initial conditions used in the envelope calculation. The calculated major radii follow the pattern of the measured values, especially near the entrance of the channel. Two frequencies are evident at first, with the mismatch amplitude being dominated by the lower frequency mode. The initial amplitude and phase of the oscillations in the measured radii are very similar to those of the calculation, but a frequency shift and significant damping are evident downstream. The envelope model assumes a constant beam emittance, with no damping or phase-mixing of the mismatch oscillation.

Detailed matrhing decreases the mismatch oscillation amplitude, as shown in Fig. 3 (same scale as Fig. 2). The remaining mismatch seems to be dominated by a high fre- 


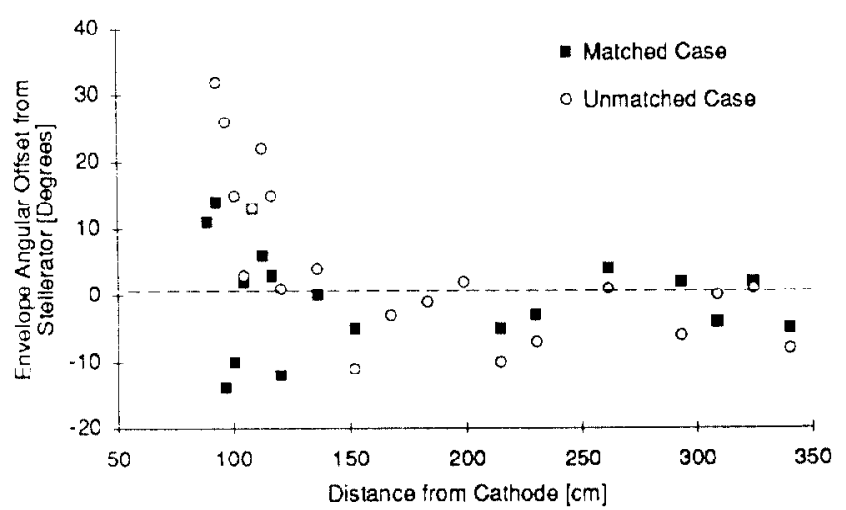

Figure 4: Orientation of the beam profile relative to the local stellarator frame.

quency mode, in contrast to the unmatched case. The beam is relatively quiescent downstream, with a measured major radius approximately $10 \%$ below the RMS radius expected from the (well known) beam current. This indicates that the edge radius we quote is probably systematically smaller than the true RMS radius, as noted above for the mismatched beam. For both configurations, the beam radii settle near the same values, dominated by the current of the beam rather than the emittance.

The orientation of the envelope ellipse relative to the local stellarator frame is shown in Figure 4, including the calculated orientation of the envelope. The deviation from the local twisted quadrupole frame is reduced significantly by matching. The envelope damping is quite rapid, with a scale length of on the order of 1 meter $(5$ cyclotron periods, 1.5 helix periods), and should be accompanied by an increased emittance. However, our measurements of the beam emittance at the exit of the long solenoid in the two cases are not significantly different. This is probably because our present optical diagnostic techniques do not include low-level regions of phase space.

In a report on a series of measurements of mismatchdriven cmittance growth in a spacc-charge dominated beam of heavy ions $[5]$, the growth was due to the development of a low-level halo of particles in phase space. Two slit emittance measurements using direct electrical pickup of the particles had a dynamic range of greater than 200:1. The evolution of a matched beam was compared against that of a beam with a large symmetric mismatch ( $x$ and $y$ oscillations in phase) and a beam with a large antysymmetric mismatch ( $x$ and $y$ out of phase). In the symmetric case, only slight envelope damping was seen over $\sim 6$ zerocurrent betatron oscillation periods, with no significant growth in emittance. The antisymmetric mismatch was strongly damped, on a scale length of less than 2.5 zerocurrent betatron periods, and the RMS ernittance grew by a factor of approximately four. Most of the increase came from regions of phase space with an intensity of less than $5 \%$ of the central peak. The peak phase space intensity dropped by only $25 \%$ with respect to that of the other beams. Such behavior would not have been resolved in our measurements to date.

\section{Conclusions}

Our present emittance measurements indicate that the central phase space density of the beam is not greatly affected by mismatches. However, the observed dispersion of the mismatch oscillations is inconsistent with zero emittance growth. As we improve our diagnostics, we should be able to resolve this anomaly. The planned two-pass SLIA system has six guide field/stellarator transitions. If each uncompensated transition caused only a $10 \%$ increase in the radial excursion of the beam, emittance growth to three times the original emittance could result, depending upon how thoroughly the mismatch were dissipated between transitions. Given the envelope damping results, it seems prudent to maintain phase space matching to avoid a large increase in the RMS emittance. Restoring the beam to a circular cross-section for guide field transport between stellarator bends also allows arbitrary choice of the phase of subsequent stellarator fields. Because of the bend, this phase is fixed when the magnet is fabricated.

\section{References}

[1] Putnam, Sidney D., "Survey of compact high current electron accelerators," in Proc. 1987 Part. Acc. Conf. March 16-19, Washington, D. C., pp. 887-891.

[2] Gluckstern, R. L., "Focusing of high current beams in continuously rotated quadrupole systems," in Proc. 1979 Lin. Acc. Conf., Brookhaven National Laboratory. BNL-51134, pp. 245-248.

[3] Roberson, C. W., A. Mondelli, and D. Chernin Phys. Rev. Lett., 50, 1983. also Part. Accel., 17:79, 1985.

[4] Anderson, O. A. and L. Soroka, "Emittance growth in intense mismatched beams," in Proc. 1987 Part. Acc. Conf. March 16-19, Washington, D. C., pp. 1043 1045 .

[5] Tiefenback, Michael G., "Experimental measurements of emittance growth in mismatched space-chargedominated beams," in Proc. 1987 Part. Acc. Conf. March 16-19, Washington, D. C., pp. 1046-1048.

[6] Kapchinskij, I. M. and V. V. Vladimirskij, "Limitations of proton bcam current in a strong focusing linear accelarator associated with the beam space charge," in Proc. Int. Conf. on High Energy Accel. and Inst., pp. 275-288, CERN, 1959.

[7] Chernin, D., "Self-consistent treat ment of equilibrium space charge effects in the $\mathrm{l}=2$ stellatron," in Proc. 1985 Part. Acc Conf. May 13-16, Vancouver, BC, Canada, IEEE Trans. on Nuc. Sci., NS-32(5), 2504 2506.

[8] Chernin, D., "Evolution of rms beam envelopes in transport systems with linear $x-y$ coupling," Part. Accel., 24(1), 1988

[9] Tiefenback, M. G. November 1989. unpublished presentation to Spiral Line Induction Accelerator working group.

[10] Lidestri, J. P., et al. proceedings of this conference. 